\title{
Beat-by-Beat Quantification of Cardiac Cycle Events Detected From Three-Dimensional Precordial Acceleration Signals
}

\section{Paukkunen, Mikko}

2016-03

Paukkunen, M , Parkkila , P , Hurnanen , T , Pankaala , M , Koivisto , T , Nieminen , T , Kettunen, R \& Sepponen, R 2016 , ' Beat-by-Beat Quantification of Cardiac Cycle Events Detected From Three-Dimensional Precordial Acceleration Signals ' , IEEE Journal of Biomedical and Health Informatics , vol. 20 , no. 2 , pp. 435-439 . https://doi.org/10.1109/JBHI.2015.2391437

http://hdl.handle.net/10138/161274

https://doi.org/10.1109/JBHI.2015.2391437

publishedVersion

Downloaded from Helda, University of Helsinki institutional repository.

This is an electronic reprint of the original article.

This reprint may differ from the original in pagination and typographic detail.

Please cite the original version. 


\title{
Beat-by-Beat Quantification of Cardiac Cycle Events Detected From Three-Dimensional Precordial Acceleration Signals
}

\author{
Mikko Paukkunen, Petteri Parkkila, Tero Hurnanen, Mikko Pänkäälä, Tero Koivisto, Tuomo Nieminen, \\ Raimo Kettunen, and Raimo Sepponen
}

\begin{abstract}
The vibrations produced by the cardiovascular system that are coupled to the precordium can be noninvasively detected using accelerometers. This technique is called seismocardiography. Although clinical applications have been proposed for seismocardiography, the physiology underlying the signal is still not clear. The relationship of seismocardiograms of on the backto-front axis and cardiac events is fairly well known. However, the 3-D seismocardiograms detectable with modern accelerometers have not been quantified in terms of cardiac cycle events. A major reason for this might be the degree of intersubject variability observed in 3-D seismocardiograms. We present a method to quantify 3-D seismocardiography in terms of cardiac cycle events. First, cardiac cycle events are identified from the seismocardiograms, and then, assigned a number based on the location in which the corresponding event was found. 396 cardiac cycle events from 9 healthy subjects and $\mathbf{1 2 0}$ cardiac cycle events from patients suffering from atrial flutter were analyzed. Despite the weak intersubject correlation of the waveforms $(0.05,0.27$, and 0.15 for the $x-$, $y$-, and $z$-axes, respectively), the present method managed to find latent similarities in the seismocardiograms of healthy subjects. We observed that in healthy subjects the distribution of cardiac cycle event coordinates was centered on specific locations. These locations were different in patients with atrial flutter. The results suggest that spatial distribution of seismocardiographic cardiac cycle events might be used to discriminate healthy individuals and those with a failing heart.
\end{abstract}

Index Terms-Accelerometer, atrial flutter, ballistocardiography, biomedical signal processing, seismocardiography (SCG).

\section{INTRODUCTION}

$\mathbf{T}$ HE cardiovascular system produces vibrations that are coupled to the precordium. These vibrations can be detected either by using palpation or auscultation, or by using

Manuscript received August 22, 2014; revised November 18, 2014 and January 5, 2015; accepted January 12, 2015. Date of current version March 3, 2016. This work was supported in part by the Finnish Cultural Foundation and The Finnish Society of Electronics Engineers.

M. Paukkunen, P. Parkkila, and R. Sepponen are with the Department of Electrical Engineering and Automation, Aalto University School of Electrical Engineering, 02150 Espoo, Finland (e-mail: mikko.paukkunen@aalto.fi; petteri.parkkila@aalto.fi; raimo.sepponen@aalto.fi).

T. Hurnanen, M. Pänkäälä, and T. Koivisto are with the Technology Research Center, University of Turku, 20520 Turku, Finland (e-mail: tero. hurnanen@utu.fi; mtpank@utu.fi; tejuko@utu.fi).

T. Nieminen is with the University of Helsinki, 00100 Helsinki, Finland and also with the South Karelia Central Hospital, 53130 Lappeenranta, Finland (e-mail: tuomo.nieminen@iki.fi).

R. Kettunen is with the University of Eastern Finland School of Medicine, 70211 Kuopio, Finland (e-mail: raimo.kettunen@ fimnet.fi).

Color versions of one or more of the figures in this paper are available online at http://ieeexplore.ieee.org.

Digital Object Identifier 10.1109/JBHI.2015.2391437 electronic sensors. The recording of precordial vibrations using accelerometers is often called seismocardiography (SCG) [1]. The research of Baevsky and Bozhenko [2], [3] are regarded as pioneering works in the field of SCG, whereas the work of Zanetti and Salerno [1] may be viewed as the revival of the field. SCG has multiple proposed clinical applications, such as ischemic heart disease detection and gating of cardiac imaging and therapy modalities [4]-[7]. The recent advances in accelerometer technology have brought upon a new generation of SCG measurement systems that measure the SCG signal in three dimensions. The 3-D SCG measurements can be implemented using individual orthogonally mounted high-sensitivity accelerometers [8] or smaller but less sensitive accelerometers that use only one integrated circuit to implement the 3-D measurement [9]-[11].

The relationship of cardiac-induced vibrations and cardiac events are fairly well known in terms of 1-D SCG [12], [13]. However, this is not the case in 3-D seismocardiograms. Although the general importance of 3-D measurements in cardiac vibration studies is widely acknowledged [10], [14]-[16], very few reports have focused on quantifying the physiological events that are manifested in 3-D seismocardiograms. The main reason for this might be that the intersubject variability of 3-D seismocardiograms is so significant. To advance SCG from a research tool to an actual clinical tool, it was necessary to be able to quantify the SCG waveforms.

This study aims to quantify 3-D SCG in terms of spatial distribution of physiological events. To study the feasibility of the proposed quantification method, ten healthy volunteers are measured and their 3-D seismocardiograms are analyzed.

\section{Materials AND MethodS}

\section{A. Equipment}

The 3-D SCG signals were measured with three orthogonally mounted accelerometers (SCA610-C21H1 A, Murata Electronics, Finland). The accelerometers and associated electronics are both described in detail in the authors' previous work [8]. The acceleration sensors had a sensitivity of $2 \mathrm{~V} / \mathrm{g}$, true dc response and rated output noise of approximately $60 \mu \mathrm{grms}$ in the frequency band of $1-50 \mathrm{~Hz}$. The accelerometers were measured at VTI's (now Murata Electronics) laboratories where they were shown to have their $-3 \mathrm{~dB}$ points at $47 \mathrm{~Hz}$. The antialiasing of the acceleration signals was done with eighth-order Bessel lowpass filters with a rated cut-off frequency of $100 \mathrm{~Hz}$ and attenuation 
of at least $96 \mathrm{~dB}$ at $800 \mathrm{~Hz}$. The Sallen-Key coupled filters were implemented using quad operational amplifiers (AD8630, Ana$\log$ Devices, USA). Changes in this particular study compared to the preceding implementation [8] were bypassing the gain stage and mounting the individual accelerometers to a smaller package than before while using eyesight to orient the sensors. The amplification setup resulted in a dc response and a gain of $0 \mathrm{~dB}$ for the acceleration measurement. Three individual accelerometers were used because, to the best of the authors' knowledge, no integrated options with similar characteristics (high sensitivity, low noise, dc response) were available during the time of implementation. Recently, integrated 3-D accelerometers with similar noise and sensitivity performance to SCG but with no dc response have been applied [17]. A dc response facilitates measuring the instantaneous inclination of the sternum which would be important in other SCG applications.

Due to the mounting process, the orthogonality of the acceleration sensors could not be guaranteed. The largest error due to mounting was measured between the $x y$ plane and the $z$-axis $\left(5^{\circ}\right)$. Thus, in the worst case, any single axis of acceleration measurement will include an error of $9 \%$.

Electrocardiography (ECG) and respiration efforts were recorded as reference signals. The ECG lead II was measured using a commercial wireless dual-lead ECG system (BC-ECG2, BIOPAC Systems Inc., Goleta, CA, USA). Respiratory efforts were recorded using a commercial wireless respiration detection system based on a respiration band (BN-RESP-XDCR, BIOPAC Systems Inc.). All data were captured using the MP150 data acquisition system (BIOPAC Systems Inc.) using a sampling rate of 1000 samples/second. The acceleration signals were coupled to the MP150 through a Universal Interface Module (UIM100 C, BIOPAC Systems Inc.). Preprocessing of the signals was done in the Acknowledgment environment (BIOPAC Systems Inc.). All postprocessing of the data was done in the MATALB environment (MATLAB R2013b, Mathworks, USA).

\section{1) In vivo Measurements:}

a) Human subject protocol: Ten male volunteers with an average weight of $79.5 \mathrm{~kg}$ (standard deviation (SD) $14.9 \mathrm{~kg}$ ), an average height of $175.2 \mathrm{~cm}(\mathrm{SD} 7.1 \mathrm{~cm})$, and an average age of 33 years (SD 8.5 years) were measured. The measurements were performed while the test subjects were in resting supine position. During the measurement, the subjects were asked to perform voluntary apnoea in order to decrease the effect of respiratory efforts on the SCG signals.

The accelerometer package was mounted on the lower part of the sternum about $1 \mathrm{~cm}$ above the xiphoid process using the double-sided adhesive tape. The orientation of the package was so that one accelerometer measured the acceleration in the backto-front ( $z$-axis), one in the right-to-left ( $x$-axis), and one in the foot-to-head direction ( $y$-axis).

All measurements of healthy individuals were performed at the Aalto University School of Electrical Engineering, Espoo, Finland, in the spirit of Helsinki declaration. A written consent was received from each subject. The contents of the test procedure and the course of its events were explained individually to the subjects before the study. The study did not contain any such intervention in the physical integrity of the test subjects,

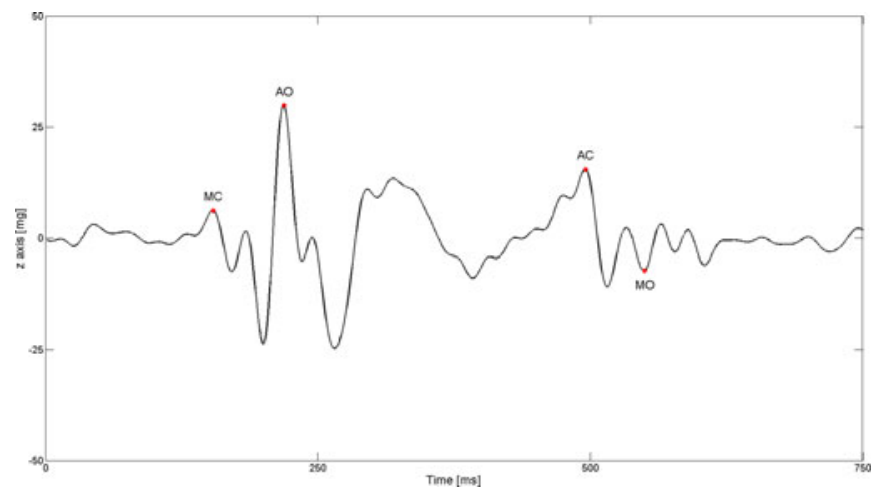

Fig. 1. Cardiac cycle events identified from the $z$-axis SCG signal.

or any other features needing an ethical review as considered by the National Advisory Board on Research Ethics in Finland.

2) Signal Processing: All signals were band-pass filtered to the range of $0.5 \mathrm{~Hz}$ to $40 \mathrm{~Hz}$. The annotation of the 3-D seismocardiograms was based on the annotation scheme proposed by Crow et al. (1994). All cardiac cycle events were detected manually from the z-axis seismocardiograms. The heartbeats were detected from the SCG signal using the peak of ECG R wave as a timing reference. The peaks of the ECG R waves were detected using the Pan-Tompkins algorithm [18] and the SCG events in the vicinity of the R peaks were investigated. Using the convention proposed by Crow et al. [12] as a general guideline, the local maximum before the beginning of the sharp downslope on the z-axis seismocardiogram following the ECG R peak was identified as mitral valve closure (MC), the positive peak after the $\mathrm{MC}$ point as aortic valve opening ( $\mathrm{AO})$, the local maximum before sharp down going slope change near the end of the ECG $\mathrm{T}$ wave as aortic valve closure (AC), and the second nadir after the $\mathrm{AC}$ point as mitral valve opening (MO). 11 consecutive heartbeats recorded during voluntary apnoea performed following inhalation were studied from each subject. Fig. 1 illustrates the cardiac cycle events identified from the $\mathrm{z}$ axis SCG signal using 11 consecutive heartbeats of a single subject. Fig. 2 shows the 3-D vector trajectory of a single heartbeat recorded from a healthy person.

\section{B. Comparison Dataset}

To investigate how disease manifests in the quantifications of cardiac cycle events, three patients (two males and one female) suffering from atrial flutter (AFl) were measured at Turku University Central Hospital. The measurements were done with an ethical permission from the ethical advisory board of The Hospital District of Southwest Finland. The weights, heights, and ages of the subjects were $108 \mathrm{~kg}, 98 \mathrm{~kg}$, and $76 \mathrm{~kg} ; 183 \mathrm{~cm}, 180 \mathrm{~cm}$, and $170 \mathrm{~cm}$; and 51 years, 57 years, and 76 years, respectively. From these subjects, ten consecutive heartbeats were recorded under resting conditions with no breathing instructions. The digital accelerometer (MMA8451Q, Freescale Semiconductor, Austin, TX, USA) used in these recordings only recorded the $z$ and $y$-axes. The signals were sampled at a rate of 800 samples/s. Digital filtering with a passband of $1-40 \mathrm{~Hz}$ was used. 


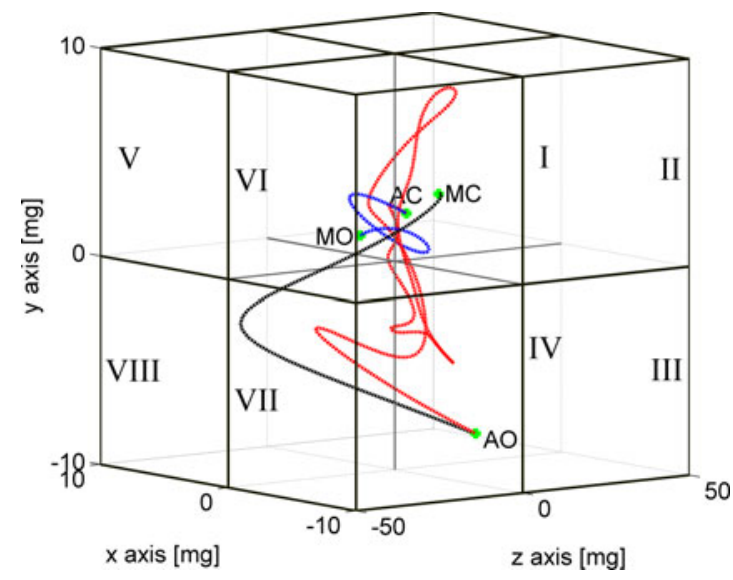

Fig. 2. 3-D vector trajectory of a single cardiac cycle. The black line depicts the time interval beginning from mitral valve closure (MC) to aortic valve opening ( $\mathrm{AO}$ ), the red line the time interval from $\mathrm{AO}$ to aortic valve closure $(\mathrm{AC})$, and the blue line the time interval from $\mathrm{AC}$ to mitral valve opening (MO).

\section{Quantification of Cardiac Cycle Events}

To quantify the spatial distribution of cardiac cycle events, the 3-D space was divided in eight rectangular octants (see Fig. 2), and each cardiac cycle event was assigned a value representing the octant in which the event was found in using the following equation:

$$
\operatorname{Octant}(x, y, z)= \begin{cases}\text { I, } & x \geq 0, y \geq 0, z \geq 0 \\ \text { II, } & x>0, y<0, z>0 \\ \text { III, } & x<0, y<0, z>0 \\ \text { IV, } & x<0, y>0, z>0 \\ \text { V, } & x>0, y>0, z<0 \\ \text { VI, } & x \geq 0, y<0, z<0 \\ \text { VII, } & x<0, y<0, z<0 \\ \text { VIII, } & x<0, y>0, z<0 .\end{cases}
$$

To quantify the variability in the morphology of the 3-D SCG waveforms squared Pearson correlation coefficients $\left(R^{2}\right)$ were computed using a window beginning from the MC point and ending at the MO point. Using this window, the intersubject correlation between waveforms was computed by correlating every obtained heartbeat with one another. The intrasubject correlation was computed similarly as the intersubject correlation but using only subject-specific heartbeats.

\section{RESULTS}

\section{A. Healthy Subjects}

Subject 4 was excluded from the analysis due to poor signal quality that prevented detecting the cardiac cycle events reliably. Thus, the total number of analysed cardiac cycles was 99 and the total number of analyzed cardiac cycle events was 396. Fig. 3 shows the location of all detected cardiac cycle events of all subjects in 3-D space.
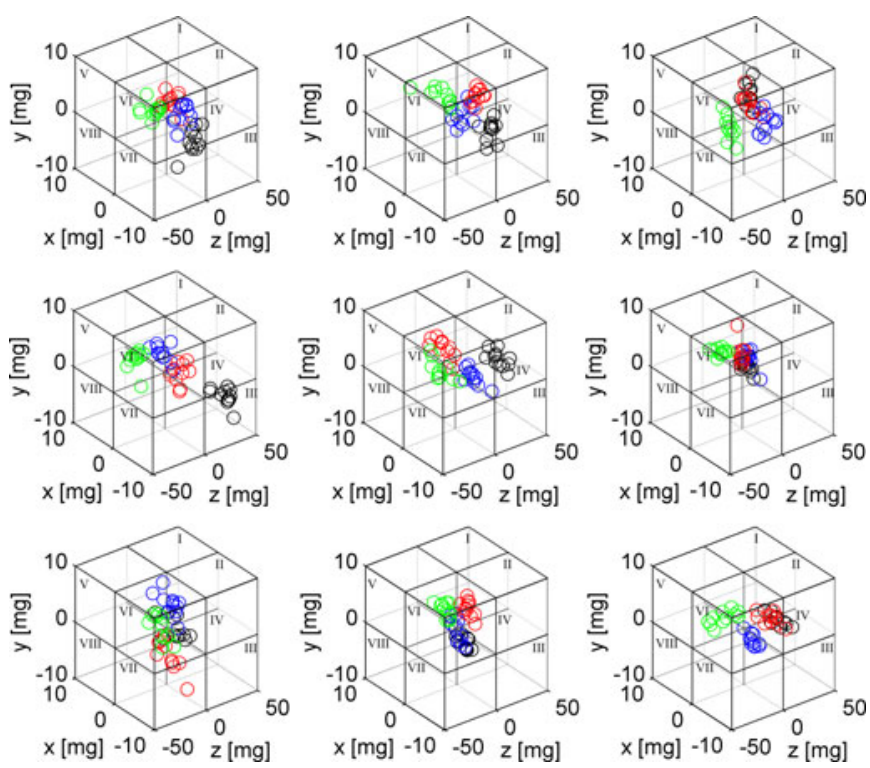

Fig. 3. All detected cardiac cycle events of all subjects. The mitral valve opening (MC) events are plotted in black, the aortic valve opening (AO) events in blue, the aortic valve closure (AC) events in red, and the mitral valve opening (MO) events in green.

TABLE I

Distribution OF ALl SUBJECTS' CARDIAC EvENTS ACROSS OCTANTS

\begin{tabular}{lcccc}
\hline \hline Octant & $\begin{array}{c}\text { MC }(\%) \\
\text { Healthy/AFl }\end{array}$ & $\begin{array}{c}\text { AO }(\%) \\
\text { Healthy/AFl }\end{array}$ & $\begin{array}{c}\text { AC(\%) } \\
\text { Healthy/AFl }\end{array}$ & $\begin{array}{c}\text { MO }(\%) \\
\text { Healthy/AFl }\end{array}$ \\
\hline I & $26 /-$ & $5 /-$ & $41 /-$ & $1 /-$ \\
II & $9 /-$ & $47 /-$ & $11 /-$ & $0 /-$ \\
III & $24 /-$ & $27 /-$ & $8 /-$ & $0 /-$ \\
IV & $30 /-$ & $20 /-$ & $38 /-$ & $0 /-$ \\
V & $2 /-$ & $0 /-$ & $0 /-$ & $55 /-$ \\
VI & $1 /-$ & $0 /-$ & $1 /-$ & $13 /-$ \\
VII & $1 /-$ & $0 /-$ & $0 /-$ & $9 /-$ \\
VIII & $6 /-$ & $0 /-$ & $0 /-$ & $21 /-$ \\
I/IV & $56 / 57$ & $25 / 53$ & $79 / 57$ & $56 / 33$ \\
II/III & $33 / 37$ & $74 / 33$ & $19 / 17$ & $0 / 10$ \\
V/VIII & $8 / 0$ & $0 / 7$ & $0 / 23$ & $76 / 33$ \\
VI/VII & $2 / 7$ & $0 / 7$ & $1 / 3$ & $22 / 23$ \\
\hline \hline
\end{tabular}

Table I shows the distribution of all identified cardiac cycle events of each analysed subject between the spatial octants. Of all identified cardiac events, $18.4 \%, 16.9 \%, 14.9 \%, 22.2 \%$, $14.4 \%, 3.8 \%, 2.5 \%$, and $6.8 \%$ were found in the octant I, II, III, IV, V, VI, VII, and VIII, respectively. On average, $25 \%$ of the cardiac cycle events detected from the 11 consecutive heartbeats of each single subject were located in a single octant, $64 \%$ in two or less octants, $94 \%$ in three or less octants, and $6 \%$ in four or more octants.

The distance of each identified cardiac cycle event from the subject-specific average spatial coordinates of the corresponding cardiac event was on average $2.5 \pm 1.1 \mathrm{mg}, 3.6 \pm$ $1.9 \mathrm{mg}, 2.3 \pm 1.0$, and $2.9 \pm 1.4 \mathrm{mg}$, for the $\mathrm{MC}, \mathrm{AO}, \mathrm{AC}$, and MO points, respectively. The mean distance of each identified cardiac event from the nearest interface of two octants was $1.1 \pm$ $0.7 \mathrm{mg}, 2.4 \pm 1 \mathrm{mg}, 1.5 \pm 0.8 \mathrm{mg}$, and $1.5 \pm 0.8$, for the MC, $\mathrm{AO}, \mathrm{AC}$, and $\mathrm{MO}$ points, respectively. 
The mean intrasubject correlation $\left(\mathrm{R}^{2}\right)$ between waveforms of all heartbeats was $0.66,0.79$, and 0.81 for the $x$-, $y$-, and $z$-axes, respectively. The mean intersubject correlation $\left(\mathrm{R}^{2}\right)$ between waveforms was $0.05,0.27$, and 0.15 for the $x$-, $-y$, and $z$-axes, respectively.

\section{B. Patients Suffering From Atrial Flutter}

Altogether 30 heartbeats were analyzed from the patients suffering from AFl. The distribution of the AFl patients' cardiac events can be found in Table I.

\section{DISCUSSION}

The intrasubject correlation of seismocardiograms was found to be reasonably strong (mean of 0.75 across all axes), which was significantly higher than in some of the previous research assessing only the $z$-axis [19]. This difference is probably mainly caused by differences between the test setups. However, the observed strong intrasubject correlation reasserts our earlier finding that our SCG measurement system produces consistent results [8]. Despite the strong intrasubject correlation, the 3-D seismocardiograms featured very weak intersubject correlation (mean of 0.16 across all axes). These observations imply that the 3-D SCG waveforms are so dissimilar between subjects that robust quantification relying only on the raw signals may not be feasible. Thus, exploring more general quantification approaches is justified. Analysis of the intrasubject distribution of cardiac event locations revealed a significant degree of clustering, which is supported by the observation that $94 \%$ of the cardiac cycle events detected from the 11 consecutive heartbeats of each individual subject were located in three or less octants. For example, all of subject 6's MC points were found in two quadrants and all of subject 6's AO, AC, and MO points were each found in a single octant. These results suggest that although the intersubject variability of 3-D seismocardiograms was weak, the 3-D seismocardiograms might still feature consistent characteristics that are similar between subjects but more advanced techniques than direct comparison of waveform morphology is needed to reveal them.

We observed that the distribution of cardiac cycle event coordinates was centred on specific octants. $73 \%$ of all cardiac events were found in octants I-IV, which is expected as all cardiac cycle events except for the MO were detected using the positive peaks in the $z$-axis acceleration signal. $96 \%$ of $\mathrm{MC}$, $\mathrm{AO}$, and $\mathrm{AC}$ points were found in octants $\mathrm{I}-\mathrm{V}$. We observed that these were distributed so that $25 \%, 23 \%, 21 \%$, and $31 \%$ of the events were located in the octants I, II, III, and IV, respectively. This indicates that while the $z$-axis consistently divided the cardiac cycle events in two halves of the 3-D space, the $y$-and $z$-axes were not as consistent, and provided less discriminatory power. Our finding seems to in part replicate the findings in [20], where Migeotte et al. observed that the $x$-axis did not reflect any physiological events. However, we found that using the present approach, the $y$-axis did not provide discriminatory power either. The lack of consistency in the $x$ - and $y$-axes signals may be explained by insufficient mechanical coupling of cardiac vibrations to the sternum, variation in anatomy of the cardiovascular system (orientation of the heart and the aortic arc), and the method used in this study to detect cardiac cycle events. The relatively strong intrasubject correlation of $x$ - and $y$-axes suggests that at least part of the cardiac vibrations in these directions are coupled to the sternum. The variation in the anatomy of the cardiovascular system might explain part of the observed intersubject variation but it should not affect the intrasubject variability of waveforms. The detection of cardiac cycle events was done using the methodology described by Crow et al. [12] as a guideline. Since the heart and blood move in three dimensions, the timing of the detected events might not be reliable using only one axis of measurement. Thus, in future research, the cardiac events should be detected with a more reliable method, such as 3-D echocardiography.

In Fig. 3, it can be seen that for some subjects, even if the location of identified cardiac cycle events is consistent, the location might be near the interface of octants. For all detected cardiac cycle events, the mean distance of each identified cardiac event from the nearest interface of two octants was smaller than the mean distance of each identified cardiac event from the subject-specific average spatial coordinates of the corresponding cardiac event. This observation strongly suggests that a more sophisticated division of the 3-D space might be more reliable in quantifying cardiac cycle events than simple division to rectangular octants. Such a division could be based on, for example, the observed spatial distribution of cardiac cycle events.

The mounting method of the acceleration sensors might have yielded estimation errors in the acceleration vector position. The severity of these errors may be negligible but the possibility of significant error must be accounted for in future research. Some portion of the orientation error might be compensated by measuring the orientation of the accelerometers and transforming the results to an orthogonal coordinate system. We suggest that in future work the accelerometers should be mounted using a repeatable process that will guarantee the orthogonality of the accelerometers.

In vectorcardiography, the changes in spatial characteristics of vectorcardiograms can be used to detect certain cardiac conditions [21], [22]. Based on the present results, a similar approach might be feasible in 3-D SCG also. Since many cardiac conditions, such as myocardial infarction or heart failure, cause changes also in the mechanical function of the heart, the observed locations of the cardiac cycle events in 3-D seismocardiograms might be a feasible method to discriminate normally functioning hearts from diseased hearts. To reassert this hypothesis, we pursued preliminary investigation of how disease manifests in the present quantifications. Table I suggests that there the coordinates of the $\mathrm{AO}, \mathrm{AC}$, and $\mathrm{MO}$ points were more evenly distributed within space in the sick patients, which is in line with the assumption that AFl introduces instability to cardiac function. To further assess the feasibility of the present method to discriminate diseased and healthy hearts, a 3-D analysis with larger sample size must be done.

\section{ACKNOWLEDGMENT}

The authors would like to thank J. Kuutti, L. Sc., for help in data collection and study design. 


\section{REFERENCES}

[1] D. Salerno and J. Zanetti, "Seismocardiography: A new technique for recording cardiac vibrations. Concept, method, and initial observations," J. Cardiovasc. Technol., vol. 9, no. 2, pp. 111-118, 1990.

[2] B. S. Bozhenko, "Seismocardiography-A new method in the study of functional conditions of the heart," Ter. Arkh., vol. 33, pp. 55-64, Sep. 1961.

[3] R. Baevsky, A. Egorov, and L. Kazarian, "Metodika seismokardiografii," Kardiologiia, vol. 18, pp. 87-89, 1964.

[4] I. Korzeniowska-Kubacka, M. Bilińska, and R. Piotrowicz, "Usefulness of seismocardiography for the diagnosis of ischemia in patients with coronary artery disease," Ann. Noninvasive Electrocardiol., vol. 10, no. 3, pp. 281287, 2005.

[5] C. Wick, J. Su, J. McClellan, O. Brand, P. Bhatti, A. Buice, A. Stillman, $\mathrm{X}$. Tang, and S. Tridandapani, "A system for seismocardiography-based identification of quiescent heart phases: Implications for cardiac imaging," IEEE Trans. Inf. Technol. Biomed., vol. 16, no. 5, pp. 869-877, Sep. 2012.

[6] K. Tavakolian, F. Khosrow-Khavar, B. Kajbafzadeh, M. Marzencki, A. Blaber, B. Kaminska, and C. Menon, "Precordial acceleration signals improve the performance of diastolic timed vibrations," Med. Eng. Phys., vol. 35, no. 8, pp. 1133-1140, 2013.

[7] M. Becker, A. Roehl, U. Siekmann, A. Koch, M. de la Fuente, R. Roissant, K. Radermacher, N. Marx, and M. Hein. (2013, Jun.). Simplified detection of myocardial ischemia by seismocardiography. Herz [Online]. Available: http://link.springer.com/article/10.1007\%2Fs00059-013-3851-x

[8] M. Paukkunen, M. Linnavuo, H. Haukilehto, and R. Sepponen, "A system for detection of three-dimensional precordial vibrations," Int. J. Meas. Technol. Instrum. Eng., vol. 2, no. 1, pp. 52-66, 2012.

[9] K. Pandia, O. T. Inan, G. T. A. Kovacs, and L. Giovangrandi, "Extracting respiratory information from seismocardiogram signals acquired on the chest using a miniature accelerometer," Physiol. Meas., vol. 33, no. 10, pp. 1643-1660, 2012

[10] P. Castiglioni, A. Faini, G. Parati, and M. Di Rienzo, "Wearable seismocardiography, Wearable Seismocardiography," in Proc. IEEE 29th Annu. Int. Conf. Eng. Med. Biol. Soc., Lyon, France, 2007, pp. 3954-3957.

[11] M. Di Rienzo, P. Meriggi, F. Rizzo, E. Vaini, A. Faini, G. Merati, G. Parati, and P. Castiglioni, "A wearable system for the seismocardiogram assessment in daily life conditions," in Proc. IEEE Annu. Int. Conf. Eng. Med. Biol. Soc., 2011, pp. 4263-4266.

[12] R. S. Crow, P. Hannan, D. Jacobs, L. Hedquist, and D. M. Salerno, "Relationship between seismocardiogram and echocardiogram for events in the cardiac cycle," Amer. J. Noninvas. Card., vol. 8, no. 1, pp. 39-46, 1994.
[13] V. Gurev, K. Tavakolian, J. Constantino, B. Kaminska, A. P. Blaber, and N. A. Trayanova, "Mechanisms underlying the isovolumic and ejection peaks in seismocardiogram morphology," J. Med. Biol. Eng.,vol. 32, no. 2, pp. 103-110, 2012.

[14] W. P. S. McKay, P. H. Gregson, B. W. S McKay, and J. Militzer, "Sternal acceleration ballistocardiography and arterial pressure wave analysis to determine stroke volume," Clin. Invest. Med., vol. 22, no. 1, pp. 4-14, 1999.

[15] P. Migeotte, S. De Ridder, J. Tank, N. Pattyn, I. Funtova, R. Baevsky, X. Neyt, and G. K. Prisk, "Three dimensional ballisto-and seismocardiography, HIJ wave amplitudes are poorly correlated to maximal systolic force vector," in Proc. IEEE Annu. Int. Conf. Eng. Med. Biol. Soc., San Diego, CA, USA, pp. 5046-5049.

[16] S. De Ridder, P. F. Migeotte, X. Neyt, N. Pattyn, and G. Prisk, "Threedimensional ballistocardiography in microgravity: A review of past research," in Proc. IEEE Annu. Int. Conf. Eng. Med. Biol. Soc., Boston, MA, USA, 2011, pp. 4267-4270.

[17] A. Wiens, M. Etemadi, S. Roy, L. Klein, and O. Inan, "Towards continuous, non-invasive assessment of ventricular function and hemodynamics: Wearable ballistocardiography," IEEE J. Biomed. Health Inform., vol. PP, no. 99, pp. 1, 2014.

[18] J. Pan and W. J. Tompkins, "A real-time QRS detection algorithm," IEEE Trans. Bio.Med. Eng., vol. BME-32, no. 3, pp. 230-236, Mar. 1985.

[19] K. Tavakolian, A. Vaseghi, and B. Kaminska, "Improvement of ballistocardiogram processing by inclusion of respiration information," Physiol. Meas., vol. 29, no. 7, pp. 771-781, 2008.

[20] P. F. Migeotte, J. Tank, N. Pattyn, I. Funtova, R. Baevsky, X. Neyt, and G. K. Prisk, "Three dimensional ballistocardiography: Methodology and results from microgravity and dry immersion," in Proc. IEEE Annu. Int. Conf. Eng. Med. Biol. Soc., Boston, MA, USA, 2011, pp. 4271-4274.

[21] M. Sederholm, "The origin of monitoring of acute myocardial infarction with continuous vectorcardiography," J. Electrocardiol., vol. 47, no. 4, pp. 418-424, 2014.

[22] A. Rubulis, L. Bergfeldt, R. Rydén, and J. Jensen, "Prediction of cardiovascular death and myocardial infarction by the QRS-T angle and $\mathrm{T}$ vector loop morphology after angioplasty in stable angina pectoris: An 8-year follow-up," J. Electrocardiol., vol. 43, no. 4, pp. 310-317, 2010.

Authors' photographs and biographies not available at the time of publication. 